\title{
COEFFICIENT ESTIMATES FOR THE FAMILY OF STARLIKE ANDCONVEX FUNCTIONS OF RECIPROCAL ORDER
}

\author{
Sudhananda Maharana ${ }^{1}$ and Deepak Bansal ${ }^{2}$ \\ ${ }^{1}$ Cent Univ Rajasthan \\ ${ }^{2}$ University College of Engineering and Technology, Bikaner
}

September 10, 2020

\begin{abstract}
In this article, we studied certain coefficient bounds and bounds on the third Hankel determinant for the family of starlike and convex functions of reciprocal order in the open unit disk $\mathbb{D}=\{z \in \mathbb{C}:|z|<1\}$.
\end{abstract}

\section{Introduction and Preliminaries}

Let $\mathcal{H}$ denote the family of analytic functions in the open unit disk $\mathbb{D}=\{z \in \mathbb{C}:|z|<1\}$, and $\mathcal{A}$ denote the class of functions $f \in \mathcal{H}$, such that

$$
f(z)=z+a_{2} z^{2}+a_{3} z^{3}+\ldots, \quad z \in \mathbb{D}
$$

We denote by $\mathcal{S}$, the functions $f$ in $\mathcal{A}$ that are univalent in $\mathbb{D}$.

A function $f \in \mathcal{A}$ is called starlike, if $f$ is univalent in $\mathbb{D}$ and $f(\mathbb{D})$ is a starlike domain with respect to the origin. Analytically, $f \in \mathcal{S}$ is called starlike, if and only if $\Re\left\{z f^{\prime}(z) / f(z)\right\}>0, z \in \mathbb{D}$. A function $f \in \mathcal{S}$ is called convex, if and only if $z f^{\prime}(z) \in \mathcal{S}^{*}$. The class of starlike functions and the class of convex functions are denoted respectively by $\mathcal{S}^{*}$ and $\mathcal{K}$.

Let $\mathcal{S}_{*}$ and $\mathcal{K}_{*}$, denotes the class of functions $f \in \mathcal{A}$, which are stalike of reciprocal order and convex of reciprocal order, respectively. Analytically, $f \in \mathcal{S}$ is called starlike of reciprocal order, if and only if $\Re\left\{f(z) / z f^{\prime}(z)\right\}>0, z \in \mathbb{D}$. A function $f \in \mathcal{S}$ is called convex of reciprocal order, if and only if $z f^{\prime}(z) \in \mathcal{S}_{*}$, and analytically this is represented by $\Re\left\{f^{\prime}(z) /\left(z f^{\prime}(z)\right)^{\prime}\right\}>0$. Various authors have studied the classes $\mathcal{S}_{*}$ and $\mathcal{K}_{*}$ and given some remarkable results (see e.g. (M. Arif et al., 2014; 2013; 2008; 2011)).

For $f \in \mathcal{A}$ of the form (1), $\Phi_{\lambda}(f):=a_{3}-\lambda a_{2}^{2}$ is the classical Fekete-Szegö functional. A classical problem settled by Fekete and Szegö (1933) is to find for each $\lambda \in[0,1]$ the maximum value of $\left|\Phi_{\lambda}(f)\right|$ over the function $f \in \mathcal{S}$, and they proved that

$$
\max _{f \in \mathcal{S}}\left|\Phi_{\lambda}(f)\right|=\{
$$




$$
\begin{array}{ll}
1+2 \exp \{-2 \lambda /(1-\lambda)\}, & \lambda \in[0,1), \\
1, & \lambda=1 .
\end{array}
$$

The problem of calculating the maximum of $\left|\Phi_{\lambda}(f)\right|$ for various subfamilies of $\mathcal{A}$, as well as $\lambda$ being an arbitrary real or complex number, was considered by many authors (see e.g. $(1992 ; 2011 ; 1969 ; 1987 ; 1993)$ ).

The Hankel determinant of Taylor coefficients for functions $f \in \mathcal{A}$ of the form (1), is denoted by $H_{q, n}(f)$, and is defined by

$$
H_{q, n}(f):=\mid
$$

$\begin{array}{cccc}a_{n} & a_{n+1} & \cdots & a_{n+q-1} \\ a_{n+1} & a_{n+2} & \cdots & a_{n+q} \\ \vdots & \vdots & \vdots & \vdots \\ a_{n+q-1} & a_{n+q} & \cdots & a_{n+2(q-1)}\end{array}$,

(2)

where $a_{1}=1 ; n, q \in \mathbb{N}=\{1,2, \ldots\}$. Several researchers including Noonan and Thomas (1976), Pommerenke (1966), Hayman (1968), Ehrenborg (2000), Noor (1992) and many more have studied the Hankel determinant and have given some remarkable results, which are useful, for example, in showing that a function of bounded characteristic in $\mathbb{D}$.

For $f \in \mathcal{A}$ of the form (1), $H_{2,1}(f):=\Phi_{1}(f)=a_{3}-a_{2}^{2}$ is the Fekete-Szegö functional. Furthermore, the upper bound of the second Hankel determinant $H_{2,2}(f)$ for various subclasses of $\mathcal{A}$ has been studied by many authors (see e.g. $\left(2012 ; 2013 ; 2006 ;\right.$ Article 281, 2013)). The third Hankel determinant $H_{3,1}(f)$ is defined by

$$
H_{3,1}(f):=\mid
$$

$\begin{array}{lll}a_{1} & a_{2} & a_{3} \\ a_{2} & a_{3} & a_{4} \\ a_{3} & a_{4} & a_{5}\end{array} \mid=\mathrm{a}_{3}\left(a_{2} a_{4}-a_{3}^{2}\right)-a_{4}\left(a_{4}-a_{2} a_{3}\right)+a_{5}\left(a_{3}-a_{2}^{2}\right)$

(3)

Recently, the author has studied the bounds on $\left|H_{3,1}(f)\right|$ for certain classes of analytic functions (see (2015; 2016)). In the current article, the upper bound of the initial coefficients and the bounds on $\left|H_{3,1}(f)\right|$ is being studied for the functions belongs to the classes $\mathcal{S}_{*}$ and $\mathcal{K}_{*}$ as stated above. In our study we shall need the class $\mathcal{P}$ of Carathéodory functions (1983), as defined below.

Let $\mathcal{P}$ denotes, the class of analytic functions in $\mathbb{D}$ with $\Re(p(z))>0$ of the form

$$
p(z)=1+c_{1} z+c_{2} z^{2}+\ldots, \quad z \in \mathbb{D} .
$$


It is well known (1983) that for the function $p \in \mathcal{P}$ is of the form (4), $\left|c_{n}\right| \leq 2$, for all $n \geq 1$. This inequality is sharp and the equality holds for the function $\varphi(z)=(1+z) /(1-z)$.

The power series (4) converges in $\mathbb{D}$ to a function in $\mathcal{P}$, if and only if the Toeplitz determinants

$$
\begin{gathered}
T_{n}(p)=\mid \\
p(z)=\sum_{\nu=1}^{m} \rho_{\nu} \frac{1+\epsilon_{\nu} z}{1-\epsilon_{\nu} z}, \quad m \geq 1,
\end{gathered}
$$

$\begin{array}{cccccc}2 & c_{1} & c_{2} & \cdots & c_{n} & \\ c_{-1} & 2 & c_{1} & \cdots & c_{n-1} & \\ c_{-2} & c_{-1} & 2 & \cdots & c_{n-2} & , \quad n \in \mathbb{N} \\ \vdots & \vdots & \vdots & \ddots & \vdots & \\ c_{-n} & c_{-n+1} & c_{-n+2} & \cdots & 2 & \end{array}$

and $c_{-n}=\bar{c}_{n}$, are all nonnegative. The only exception is when $p(z)$ has the form where $\rho_{\nu}>0,\left|\epsilon_{\nu}\right|=1$, and $\epsilon_{k} \neq \epsilon_{l}$ if $k \neq l ; k, l=1,2, \cdots, m$; we have then $T_{n}(p)>0$ for $n<m-1$ and $T_{n}(p)=0$ for $n \geq m$. This necessary and sufficient condition is due to Carathéodory and Toeplitz and can be found in (1958). In particular, for $n=2$, we have

$$
T_{2}(p)=\mid
$$

$\begin{array}{ccc}2 & c_{1} & c_{2} \\ \bar{c}_{1} & 2 & c_{1} \\ \bar{c}_{2} & \bar{c}_{1} & 2\end{array}=8+2 \Re\left\{c_{1}^{2} \bar{c}_{2}\right\}-2\left|c_{2}\right|^{2}-4\left|c_{1}\right|^{2} \geq 0$,

which is equivalent to

$$
2 c_{2}=c_{1}^{2}+x\left(4-c_{1}^{2}\right)
$$

for some $x$ with $|x| \leq 1$. Similarly, if

$$
T_{3}(p)=\mid
$$

$\begin{array}{cccc}2 & c_{1} & c_{2} & c_{3} \\ \bar{c}_{1} & 2 & c_{1} & c_{2} \\ \bar{c}_{2} & \bar{c}_{1} & 2 & c_{1} \\ \bar{c}_{3} & \bar{c}_{2} & \bar{c}_{1} & 2\end{array}$


then $T_{3}(p) \geq 0$ is equivalent to

$$
\left|\left(4 c_{3}-4 c_{1} c_{2}+c_{1}^{3}\right)\left(4-c_{1}^{2}\right)+c_{1}\left(2 c_{2}-c_{1}^{2}\right)^{2}\right| \leq 2\left(4-c_{1}^{2}\right)^{2}-2\left|\left(2 c_{2}-c_{1}^{2}\right)\right|^{2} .
$$

Solving (6) with the help of (5), we get

$$
4 c_{3}=c_{1}^{3}+2 c_{1} x\left(4-c_{1}^{2}\right)-c_{1} x^{2}\left(4-c_{1}^{2}\right)+2\left(4-c_{1}^{2}\right)\left(1-|x|^{2}\right) z,
$$

for some $x$ and $z$ with $|x| \leq 1$ and $|z| \leq 1$. Furthermore, the following well-known results are being useful to obtain our main results.

Lemma 1. (1969) If $p \in \mathcal{P}$, then for any complex number $\nu$,

$$
\left|c_{2}-\nu c_{1}^{2}\right| \leq 2 \max \{1,|2 \nu-1|\},
$$

and equality holds for the functions given by

$$
\psi(z)=\frac{1+z^{2}}{1-z^{2}} \text { and } \varphi(z)=\frac{1+z}{1-z} .
$$

Lemma 2. (1969) Let the function $p$ given by (4) is in the class $\mathcal{P}$. Then for all $n$ and $s(1 \leq s<n)$, we have $\left|c_{n}-c_{s} c_{n-s}\right| \leq 2$.

Lemma 3. (1958) (See also (1982)) If $p \in \mathcal{P}$, then the following expressions are all bounded by 2, and are all sharp:

1. $\left|c_{1}^{2}-c_{2}\right|$,

2. $\left|c_{3}-2 c_{1} c_{3}+c_{3}\right|$,

3. $\left|c_{1}^{4}+2 c_{1} c_{3}+c_{2}^{2}-3 c_{1}^{2} c_{2}-c_{4}\right|$,

4. $\left|c_{1}^{5}+3 c_{1} c_{2}^{2}+3 c_{1}^{2} c_{3}-4 c_{1}^{3} c_{2}-2 c_{1} c_{4}-2 c_{2} c_{3}+c_{5}\right|$

5. $\left|c_{1}^{6}+6 c_{1}^{2} c_{2}^{2}+4 c_{1}^{3} c_{3}+2 c_{1} c_{5}+2 c_{2} c_{4}+c_{3}^{2}-c_{2}^{3}-5 c_{1}^{4} c_{2}-3 c_{1}^{2} c_{4}-6 c_{1} c_{2} c_{3}-c_{6}\right|$.

The following inequalities can also be obtained in the proof of a result in (1982)

a. $\left|2 c_{1}^{2}-c_{2}\right| \leq 6$

b. $\left|-6 c_{1}^{3}+7 c_{1} c_{2}-2 c_{3}\right| \leq 24$

c. $\left|24 c_{1}^{4}-46 c_{1}^{2} c_{2}+22 c_{1} c_{3}+7 c_{2}^{2}-6 c_{4}\right| \leq 120$

d. $\left|-120 c_{1}^{5}+96 c_{4} c_{1}+50 c_{2} c_{3}+326 c_{1}^{3} c_{2}-202 c_{1}^{2} c_{3}-127 c_{1} c_{2}^{2}-24 c_{5}\right| \leq 720$. 
Lemma 4. (2015) Let $p \in \mathcal{P}$. Then for all $n, m \in \mathbb{N}$,

$$
\left|\mu c_{n} c_{m}-c_{m+n}\right| \leq\{
$$

$2, \quad \mu \in[0,1]$,

$2|2 \mu-1|$, elsewhere.

If $0<\mu<1$, then the inequality is sharp for the function $p(z)=\left(1+z^{m+n}\right) /\left(1-z^{m+n}\right)$. In other cases, the inequality is sharp for the function $p(z)=(1+z) /(1-z)$.

\section{$H_{3,1}(f)$ for the function belongs to the class $\mathcal{S}_{*}$}

The following is our first result in this section.

Theorem 0.1. Let the function $f$ given by (1) is in the class $\mathcal{S}_{*}$. Then we have $\left|a_{n}\right| \leq n, n=2,3,4$. This result is sharp and equality is attained for the function $e_{1}(z)=z(1+z)^{-2}$.

Proof. Let us consider $f \in \mathcal{S}_{*}$. Then by the definition, we have

$$
f(z)=z f^{\prime}(z) p(z)
$$

where $p \in \mathcal{P}$ is of the form (4). Substituting the series expansion of $f(z), f^{\prime}(z)$ and $p(z)$ in (1), and equating the coefficients, we get

$$
a_{n}=\frac{1}{1-n}\left(c_{n-1}+2 a_{2} c_{n-2}+3 a_{3} c_{n-3}+\cdots+(n-1) a_{n-1} c_{1}\right),
$$

which in particular gives us

$-\mathrm{a}_{3}\left|=\frac{1}{4}\right| 3 c_{1}^{2}-x\left(4-c_{1}^{2}\right) \mid$

$-\mathrm{a}_{4}\left|=\frac{1}{12}\right|-6 c_{1}^{3}+\left(4-c_{1}^{2}\right)\left\{5 c_{1} x+c_{1} x^{2}-2\left(1-|x|^{2}\right) z\right\} \mid$. 
$a_{2}=-c_{1}, a_{3}=\frac{1}{2}\left(2 c_{1}^{2}-c_{2}\right), a_{4}=\frac{1}{6}\left(7 c_{1} c_{2}-2 c_{3}-6 c_{1}^{3}\right)$,

and

$a_{5}=\frac{1}{24}\left(24 c_{1}^{4}-46 c_{1}^{2} c_{2}+20 c_{1} c_{3}+9 c_{2}^{2}-6 c_{4}\right)$.

Bounds for $\left|a_{2}\right|$ is obvious as $\left|c_{1}\right| \leq 2$. Bounds for $\left|a_{3}\right|$ and $\left|a_{4}\right|$ can be directly obtained from results mentioned in $a$ and $b$ of Lemma 3. Furthermore, by using (5) and (7) in (??), for some $x$ and $z$ such that $|x| \leq 1$ and $|z| \leq 1$, we obtain

and

To show the sharpness, by setting $c_{1}=2$ and $x=1$ in (5) and (7), we obtain $c_{2}=c_{3}=2$. Using these values in the above relations, we find that the result is sharp and the extremal function would be $e_{1}(z)=z(1+z)^{-2}$. This completes the proof of the theorem.

Theorem 0.2. Let the function $f$ given by (1) is in the class $\mathcal{S}_{*}$. Then we have $\left|a_{5}\right| \leq 39 / 7$.

Proof. If $f \in \mathcal{S}_{*}$, then by using the value of $a_{5}$ from (??), we obtain

$-\mathrm{a}_{5}\left|=\frac{1}{96}\right| 27 c_{1}^{4}+\left(4-c_{1}^{2}\right)\left\{-46 c_{1}^{2} x-23 c_{1}^{2} x^{2}+28 c_{1}\left(1-|x|^{2}\right) z+36 x^{2}\right\}-24\left(c_{4}-c_{1} c_{3}\right) \mid$.

$-\mathrm{a}_{5} \mid \leq \frac{1}{96}\left[27 c^{4}+\left(4-c^{2}\right)\left\{46 c^{2} \mu+23 c^{2} \mu^{2}+28 c\left(1-\mu^{2}\right)+36 \mu^{2}\right\}+48\right]:=D(c, \mu)$.

$\partial D \frac{1}{\partial \mu=\frac{1}{48}\left[\left(4-c^{2}\right)\left\{23 c^{2}(1+\mu)+4 \mu(9-7 c)\right\}\right]}$.

$\max _{\mu \in[0,1]} D(c, \mu)=D(c, 1)=\frac{1}{16}\left(-7 c^{4}+40 c^{2}+32\right)$. 


$$
\left|a_{5}\right|=\frac{1}{24}\left|24 c_{1}^{4}-46 c_{1}^{2} c_{2}+14 c_{1} c_{3}+9 c_{2}^{2}-6\left(c_{4}-c_{1} c_{3}\right)\right|
$$

By using (5) and (7), for some $x$ and $z$ such that $|x| \leq 1$ and $|z| \leq 1$, we get

If $p(z) \in \mathcal{P}$, then $p\left(e^{i \alpha} z\right) \in \mathcal{P}$. We can always select a real $\alpha$ in $p\left(e^{i \alpha} z\right)$ so that $c_{n} e^{i \alpha n} \geq 0$. Hence we may suppose that $c_{n} \geq 0(n \in \mathbb{N})$. Furthermore, the power series (4) converges in $\mathbb{D}$ to a function in $\mathcal{P}$, if and only if the Toeplitz determinants $T_{n}(p)$ and $c_{-n}=\bar{c}_{n}$, are all nonnegative, i.e. $c_{1}$ is real, $c_{1} \geq 0$ and $\left|c_{1}\right| \leq 2$. Therefore, letting $c_{1}=c$, we may assume without restriction that $c \in[0,2]$. Hence, applying the triangle inequality with $\mu=|x|$, and applying Lemma 2, we obtain

Now we need to maximize $D(c, \mu)$ on the region $\Omega=\{(c, \mu): 0 \leq c \leq 2$ and $0 \leq \mu \leq 1\}$. For this, first we estimate

For $0<\mu<1$, and for fixed $c$ with $0<c<2$, we observe that $\frac{\partial D}{\partial \mu}>0$. Therefore, $D(c, \mu)$ becomes an increasing function of $\mu$, and hence it cannot have a maximum value at any point in the interior of the closed region $\Omega$. Moreover, for a fixed $c \in[0,2]$, we have

Therefore, by the second derivative test we can see that $D(c, 1)$ has maximum value at $c$, where $c^{2}=20 / 7$.

$\partial D \overline{\partial c=\frac{1}{48}\left[54 c^{3}+23 c \mu\left(4-c^{2}\right)(2+\mu)-c\left(46 c^{2} \mu+23 c^{2} \mu^{2}+36 \mu^{2}\right)+14\left(1-\mu^{2}\right)\left(4-3 c^{2}\right)\right]}$.

Furthermore, if we look for the critical points on the boundary of $\Omega$, we estimate

Now we look for the critical point of $D(c, \mu)$ which must satisfy $\frac{\partial D}{\partial \mu}=0$ and $\frac{\partial D}{\partial c}=0$, and one can check easily that the points $(c, \mu)$ satisfying such conditions are not interior point of $\Omega$. So the maximum cannot attain in the interior of $\Omega$. Now to see on the boundary, taking the boundary line $L_{1}=\{(2, \mu): 0 \leq \mu \leq 1\}$, we have $D(2, \mu)=5$ which is a constant. Along $L_{2}=\{(0, \mu): 0 \leq \mu \leq 1\}$, we have $D(0, \mu)=\left(1+3 \mu^{2}\right) / 2$, which gives the critical point $(0,0)$. Along $L_{3}=\{(c, 1): 0 \leq c \leq 2\}$, we have $D(c, 1)=\left(-7 c^{4}+40 c^{2}+32\right) / 16$, which gives the critical points $(0,1)$ and $(\sqrt{20 / 7}, 1)$. Along $L_{4}=\{(c, 0): 0 \leq c \leq 2\}$, we have $D(c, 0)=$ $\left(27 c^{4}-28 c^{3}+112 c+48\right) / 96$, which gives no critical points in $\Omega$. Observe that

$$
D(0,0)<D(0,1)<D(2, \mu)<D(\sqrt{20 / 7}, 1) .
$$

Hence

$$
\max _{\Omega} D(c, \mu)=D(\sqrt{20 / 7}, 1)=39 / 7 .
$$

This completes the proof.

Remark. For $f \in \mathcal{S}_{*}$ of the form (1), Arif et al. (M. Arif et al., 2014) obtained that

$$
\left|a_{2}\right| \leq 2 \quad \text { and } \quad\left|a_{n}\right| \leq \frac{2}{n-1} \prod_{k=2}^{n-1}\left(\frac{3 k-1}{k-1}\right) \quad(n=3,4,5, \cdots) .
$$

Here we observe that, our result obtained in Theorem 0.1 and Theorem 0.2 provides the improvement in the upper bound of the initial coefficients $a_{n}, n=3,4,5$. 
Theorem 0.3. Let the function $f$ given by $(1)$ is in the class $\mathcal{S}_{*}$. Then we have

$$
\left|a_{3}-a_{2}^{2}\right| \leq 1, \quad\left|a_{2} a_{3}-a_{4}\right| \leq 2 \quad \text { and } \quad\left|a_{2} a_{4}-a_{3}^{2}\right| \leq 1
$$

These inequalities in (3) are sharp and the extremal function is $e_{1}(z)=z(1+z)^{-2}$.

$-\mathrm{a}_{3}-a_{2}^{2}|=| \frac{-c_{2}}{2}|, \quad| a_{2} a_{3}-a_{4}\left|=\frac{1}{3}\right|-2 c_{1} c_{2}+c_{3} \mid$

$-\mathrm{a}_{2} a_{4}-a_{3}^{2}\left|=\frac{1}{24}\right|-4 c_{1}^{2} c_{2}+8 c_{1} c_{3}-6 c_{2}^{2} \mid$

Proof. If $f \in \mathcal{S}_{*}$, then the values of $a_{2}, a_{3}$ and $a_{4}$ are given in (??). Using these values, we obtain and

Clearly, it follows that $\left|a_{3}-a_{2}^{2}\right|=\left|c_{2} / 2\right| \leq 1$.

$-\mathrm{a}_{2} a_{3}-a_{4}\left|\leq \frac{1}{3}\right| 2 c_{1} c_{2}-c_{3} \mid \leq \frac{1}{3}[2|2 \cdot 2-1|]=2$.

Now, by using Lemma 4, we obtain

Furthermore, by using (5) and (7), for some $x$ and $z$ such that $|x| \leq 1$ and $|z| \leq 1$, we get

$-\mathrm{a}_{2} a_{4}-a_{3}^{2} \mid \leq \frac{1}{48}\left[\left(4-c^{2}\right)\left\{8 c+\left(c^{2}-8 c+12\right) \mu^{2}+2 c^{2} \mu\right\}+3 c^{4}\right]:=F_{3}(c, \mu)$.

$\left|a_{2} a_{4}-a_{3}^{2}\right|=\frac{1}{48}\left|\left(4-c_{1}^{2}\right)\left[-2 c_{1}^{2} x-4 c_{1}^{2} x^{2}-3 x^{2}\left(4-c_{1}^{2}\right)+8 c_{1}\left(1-|x|^{2}\right) z\right]-3 c_{1}^{4}\right|$.

(4) 
As $\left|c_{1}\right| \leq 2$, letting $c_{1}=c$, we may assume without restriction that $c \in[0,2]$. Thus, applying the triangle inequality with $\mu=|x|$, we obtain

Next, by differentiating $F_{3}$ with respect to $\mu$, we observe that, $F_{3}$ is an increasing function of $\mu$ on $[0,1]$. Thus it attains the maximum value at $\mu=1$. Again $F_{3}(c, 1)=1$, is a constant. Hence

$$
\max _{\Omega} F_{3}(c, \mu)=F_{3}(c, 1)=1 .
$$

To get the sharpness, by setting $c_{1}=2$ and $x=1$ in (5) and (7), we obtain $c_{2}=c_{3}=2$. Using these values, we get the results in (1) are sharp and the extremal function would be $e_{1}(z)=z(1+z)^{-2}$. This completes the proof of the theorem.

Theorem 0.4. Let the function $f$ given by $(1)$ is in the class $\mathcal{S}_{*}$. Then we have

$$
\left|H_{3,1}(f)\right| \leq \frac{116}{7}
$$

$-\mathrm{H}_{3,1}(f)|\leq| a_{3}|| a_{2} a_{4}-a_{3}^{2}|+| a_{4}|| a_{2} a_{3}-a_{4}|+| a_{5}|| a_{3}-a_{2}^{2} \mid \leq 3+8+\frac{39}{7}=\frac{116}{7}$,

Proof. Using the bounds obtained above in Theorem 0.1-Theorem 0.3 and applying the triangle inequality, we estimate

and this completes the proof.

\section{$H_{3,1}(f)$ for the function belongs to the class $\mathcal{K}_{*}$}

Theorem 0.5. Let the function $f$ given by (1) is in the class $\mathcal{K}_{*}$. Then we have $\left|a_{n}\right| \leq 1, n=2,3,4$.

Proof. Let $f \in \mathcal{K}_{*}$, then by the hypothesis it is clear that $f(z) \in \mathcal{K}_{*}$ if and only if $z f^{\prime}(z) \in \mathcal{S}_{*}$. Thus replacing $a_{n}$ by $n a_{n}$ in (??), we obtain

$a_{2}=-\frac{1}{2} c_{1}, a_{3}=\frac{1}{6}\left(2 c_{1}^{2}-c_{2}\right), a_{4}=\frac{1}{24}\left(7 c_{1} c_{2}-2 c_{3}-6 c_{1}^{3}\right)$,

and

$a_{5}=\frac{1}{120}\left(24 c_{1}^{4}-46 c_{1}^{2} c_{2}+20 c_{1} c_{3}+9 c_{2}^{2}-6 c_{4}\right)$. 
Bounds for $\left|a_{2}\right|$ is obvious as $\left|c_{1}\right| \leq 2$. Bounds for $\left|a_{3}\right|$ and $\left|a_{4}\right|$ can be directly obtained from results mentioned in $a$ and $b$ of Lemma 3 . This completes the proof of the theorem.

Theorem 0.6. Let the function $f$ given by (1) is in the class $\mathcal{K}_{*}$. Then we have $\left|a_{5}\right| \leq 39 / 35$.

Proof. Let $f \in \mathcal{K}_{*}$, then using $a_{5}$ from (1), we can write

$-\mathrm{a}_{5}\left|=\frac{1}{480}\right| 27 c_{1}^{4}+\left(4-c_{1}^{2}\right)\left\{-46 c_{1}^{2} x-23 c_{1}^{2} x^{2}+28 c_{1}\left(1-|x|^{2}\right) z+36 x^{2}\right\}-24\left(c_{4}-c_{1} c_{3}\right) \mid$.

$-\mathrm{a}_{5} \mid \leq \frac{1}{480}\left[27 c^{4}+\left(4-c^{2}\right)\left\{46 c^{2} \mu+23 c^{2} \mu^{2}+28 c\left(1-\mu^{2}\right)+36 \mu^{2}\right\}+48\right]:=Z(c, \mu)$.

$\partial Z \overline{\partial \mu=\frac{1}{480}\left[\left(4-c^{2}\right)\left\{23 c^{2}(1+\mu)+4 \mu(9-7 c)\right\}\right]>0}$ for $\quad(0 \leq \mu \leq 1)$.

$$
\left|a_{5}\right|=\frac{1}{120}\left|24 c_{1}^{4}-46 c_{1}^{2} c_{2}+14 c_{1} c_{3}+9 c_{2}^{2}-6\left(c_{4}-c_{1} c_{3}\right)\right| .
$$

By using the relations (5) and (7), for some $x$ and $z$ such that $|x| \leq 1$ and $|z| \leq 1$, we estimate

As $\left|c_{1}\right| \leq 2$, letting $c_{1}=c$, we may assume without restriction that $c \in[0,2]$. Thus applying the triangle inequality and Lemma 2 with $\mu=|x|$, we obtain

Differentiating $Z(c, \mu)$ with respect to $\mu$, we get

Note that, $Z$ is an increasing function of $\mu$ on $[0,1]$. Thus it attains maximum value at $\mu=1$. Again, $Z(c, 1)=\left(-21 c^{4}+120 c^{2}+96\right) / 240$, is an increasing function of $c$ on $[0, \sqrt{20 / 7}]$. Thus $(\sqrt{20 / 7}, 1)$ is a critical point of $Z$. 
Again, if we look for the critical points on the boundary of $\Omega$, as we have done earlier, we get $(0,0),(0,1)$ and $(2, \mu), 0 \leq \mu \leq 1$ are the other critical points in $\Omega$, and for these points we have

$$
Z(0,0)<Z(0,1)<Z(2, \mu)<Z(\sqrt{20 / 7}, 1) .
$$

Hence

$$
\max _{\Omega} Z(c, \mu)=Z(\sqrt{20 / 7}, 1)=39 / 35 .
$$

This completes the proof of the theorem.

Remark. For $f \in \mathcal{K}_{*}$ of the form (1), Arif et al. (M. Arif et al., 2014) obtained that

$$
\left|a_{2}\right| \leq 1 \quad \text { and } \quad\left|a_{n}\right| \leq \frac{2}{n(n-1)} \prod_{k=2}^{n-1}\left(\frac{3 k-1}{k-1}\right) \quad(n=3,4,5, \cdots) .
$$

Here we observe that, our result obtained in Theorem 0.5 and Theorem 0.6 provides the improvement in the upper bound of the initial coefficients $a_{n}, n=3,4,5$.

Theorem 0.7. Let the function $f$ given by $(1)$ is in the class $\mathcal{K}_{*}$. Then we have

$$
\left|a_{3}-a_{2}^{2}\right| \leq \frac{1}{3}, \quad\left|a_{2} a_{3}-a_{4}\right| \leq \frac{4}{3} \quad \text { and } \quad\left|a_{2} a_{4}-a_{3}^{2}\right| \leq \frac{1}{8}
$$

The first inequality of (2) is sharp and equality is attended for the function $e_{3}(z)=z+\frac{1}{3} z^{3}$.

$-\mathrm{a}_{3}-a_{2}^{2}\left|=\frac{1}{12}\right| c_{1}^{2}-2 c_{2}|,| a_{2} a_{3}-a_{4}\left|=\frac{1}{24}\right| 2 c_{1}^{3}-5 c_{1} c_{2}+2 c_{3} \mid$,

$-\mathrm{a}_{2} a_{4}-a_{3}^{2}\left|=\frac{1}{144}\right|-5 c_{1}^{2} c_{2}+6 c_{1} c_{3}+2 c_{1}^{4}-4 c_{2}^{2} \mid$.

$-\mathrm{a}_{3}-a_{2}^{2}\left|=\frac{1}{12}\right| c_{1}^{2}-2 c_{2}\left|=\frac{1}{6}\right| c_{2}-\frac{1}{2} c_{1}^{2} \mid \leq \frac{1}{6} \cdot 2 \max \{1,|2(1 / 2)-1|\}=\frac{1}{3}$. 
Proof. If $f \in \mathcal{K}_{*}$, then by using the values of $a_{2}, a_{3}$ and $a_{4}$ which are given in (1), we obtain and

By using Lemma 1 we obtain

Next by using Lemma 4, we obtain

$$
\begin{aligned}
\left|a_{2} a_{3}-a_{4}\right|=\frac{1}{24}\left|2 c_{1}^{3}-5 c_{1} c_{2}+2 c_{3}\right| & \leq \frac{1}{24}\left[2\left|c_{1}\right|^{3}+2\left|\frac{5}{2} c_{1} c_{2}-c_{3}\right|\right] \\
& \leq \frac{1}{24}[2 \cdot 8+2 \cdot 2|2(5 / 2)-1|]=\frac{4}{3}
\end{aligned}
$$

Now, by using the relations (5) and (7), we obtain

$$
\left|a_{2} a_{4}-a_{3}^{2}\right|=\frac{1}{288}\left|\left(4-c_{1}^{2}\right)\left\{-3 c_{1}^{2} x-\left(4-c_{1}^{2}\right) 2 x^{2}+6 c_{1}\left(1-|x|^{2}\right) z-3 c_{1}^{2} x^{2}\right\}\right|
$$

As $\left|c_{1}\right| \leq 2$, letting $c_{1}=c$, we can assume without restriction that $c \in[0,2]$. Thus applying the triangle inequality with $\mu=|x|$, we get

$\partial G_{3} \frac{1}{\partial \mu=\frac{1}{288}\left(4-c^{2}\right)\left\{3 c^{2}+\left(8-6 c+c^{2}\right) 2 \mu\right\}>0}$ for $\quad 0 \leq \mu \leq 1$.

$\max _{0 \leq \mu \leq 1} G_{3}(c, \mu)=G_{3}(c, 1)=\left(8+2 c^{2}-c^{4}\right) / 72=\mathcal{G}_{3}(c)$.

$$
\left|a_{2} a_{4}-a_{3}^{2}\right| \leq \frac{1}{288}\left[\left(4-c^{2}\right)\left\{6 c+3 c^{2} \mu+\left(8-6 c+c^{2}\right) \mu^{2}\right\}\right]:=G_{3}(c, \mu)
$$

Furthermore, differentiating $G_{3}(c, \mu)$ with respect to $\mu$, we get

Hence $G_{3}(c, \mu)$ is an increasing function of $\mu$ on $[0,1]$. Thus, it attains maximum value at $\mu=1$. Let 
Again note that, $\mathcal{G}_{3}(c)$ is an increasing function on $[0,1]$, so $\mathcal{G}_{3}(c)$ attend maximum value at $c=1$. Hence $G_{3}(c, \mu)$ have maximum value at the point $(1,1)$, that is

$$
\max _{\Omega} G_{3}(c, \mu)=G_{3}(1,1)=1 / 8 \text {. }
$$

This completes the proof of the theorem.

$-\mathrm{H}_{3,1}(f) \mid \leq \frac{1537}{840}$

Theorem 0.8. Let the function $f$ given by $(1)$ is in the class $\mathcal{K}_{*}$. Then we have

$-\mathrm{H}_{3,1}(f)|\leq| a_{3}|| a_{2} a_{4}-a_{3}^{2}|+| a_{4}|| a_{2} a_{3}-a_{4}|+| a_{5}|| a_{3}-a_{2}^{2} \mid \leq \frac{1}{8}+\frac{4}{3}+\frac{39}{105}=\frac{1537}{840}$,

Proof. Using Theorem 0.5-Theorem 0.7 and applying the triangle inequality, we obtain that and this completes the proof.

\section{References}

Article Id 989640, 1-6. (2014).

(2013).

(2008).

(2011).

(1933).

(1992).

(2011).

(1969).

(1987).

(1993).

(1976).

(1966). 
(1968).

(2000).

(1992).

(2012).

(2013).

(2006).

(2013).

(2015).

(2016).

(1983).

(1958).

(1969).

(1982).

(2015). 